\title{
Encuesta a profesorado universitario sobre el déficit de atención e hiperactividad en personas jóvenes adultas
}

\author{
González, Juanita \\ Encuesta a profesorado universitario sobre el déficit de atención e hiperactividad en personas jóvenes adultas \\ Revista Educación, vol. 42, núm. 1, 2018 \\ Universidad de Costa Rica, Costa Rica \\ Disponible en: http://www.redalyc.org/articulo.oa?id=44051918011 \\ DOI: https://dx.doi.org/10.15517/revedu.v42i1.22714
}

Esta obra está bajo una Licencia Creative Commons Atribución-NoComercial-SinDerivar 3.0 Internacional. 


\title{
Encuesta a profesorado universitario sobre el déficit de atención e hiperactividad en
} personas jóvenes adultas

\author{
Teachers' Survey about the Attention Deficit Disorder and Hyperactivity in College Students
}

Juanita González [1]

Colegio Marine Academy of Science and Technology,

DOI: https://dx.doi.org/10.15517/revedu.v42i1.22714

Estados Unidos

j.gonzalez72@rocketmail.com

\section{Resumen:}

Este estudio fue realizado con el fin de evaluar y conocer el conocimiento que tiene el personal docente sobre la condición del ADD/ ADHD en jóvenes de universidad. En la investigación se identificaron los métodos de enseñanza administrados por el profesorado al detectar el ADD/ADHD en su alumnado. Se conoce que el trastorno de déficit de atención o el trastorno del déficit de atención con hiperactividad (según sus siglas en inglés ADD Y ADHD) es una condición neurológica que puede afectar el bienestar físico, social y emocional de una persona. Los síntomas más comunes que se presentan son: distracción, impulsividad e hiperactividad, y han sido relacionados con bajos resultados académicos. Debido al impacto que este déficit ejerce en la educación, es importante reconocer la condición en estudiantes del nivel superior y de esta manera dar la ayuda educativa necesaria. Se entrevistó un variado grupo de docentes que impartían clases en diferentes universidades y colegios de Puerto Rico. Se observó que una gran parte (más del 60\%) tiene conocimiento de la existencia del déficit de atención e hiperactividad en la sociedad (en la niñez). Quedó establecido que la mayoría, un 80 porciento de participntes no tiene conocimiento de que esta condición deficitaria persiste a lo largo de la vida y que el individuo puede manifestar síntomas del ADD/ADHD en su edad adulta.

Palabras clave: Trastorno de déficit de atención con hiperactividad, síntoma, niñez, educación, estudiante.

\section{ABstract:}

This study was performed to to evaluate and recognize the teachers' knowledge about the ADHD/ADD in their students. In the investigation, we identified the teachers' learning methods applied when they recognize the deficit on the students. The Attention Deficit Disorder and Hyperactivity (known as ADD and ADHD) is a neurological condition that might affect the physical, social, and emotional condition of an individual. The common symptoms that might be presents are: distraction, impulsivity and hyperactive. These symptoms have been related with academic failure. Due to the impact that this deficit has on the education, it is important to recognize the condition in college students and give them the necessary help. We interviewed a group of teachers from different universities and colleges in Puerto Rico. It was observed that a substantial part of the teacher (more than 60\%) interviewed had knowledge about the prevalence of the ADHD/ADD in the society (in children). It was established that most of the teachers don't know that the conditions persist during adulthood and adults can show symptoms of ADD/ADHD during adult hood.

KEYWORDS: attention deficit disorder with hyperactivity, education, symptoms, childhood, student.

\section{INTRODUCCIÓN}

El trastorno de déficit de atención o el trastorno del déficit de atención e hiperactividad (conocidos por sus siglas en inglés ADD Y ADHD) es una condición neurológica que puede afectar el bienestar físico, social y emocional de una persona. El diagnóstico clínico "oficial” es ADHD, dentro de este se encuentran quienes tienen un diagnóstico en donde predomina la falta de atención y quienes tienen un diagnóstico en los que

\section{NOTAS DE AUTOR}

[1] Estudiante de Doctorado en la Universidad Nacional a Distancia de Madrid, España. Profesora de Español del Colegio Marine Academy, Science and Technology. Profesora de Técnico Quirúrgico en el Colegio Start Career. Master en Educación General y Primaria. 
predomina la impulsividad e hiperactividad (Fowler, 1995; Neuwirth, 1994; Ortiz, Sáenz, Escalona, y Vargas, 1996).

Esta condición se presenta comúnmente en la niñez; pero no solo se limita a esta etapa, ya que además es un problema que generalmente persiste a lo largo de la vida. Probablemente, este desorden lo causen factores biológicos que influyen en las actividades de los neurotransmisores en ciertas partes del cerebro, A su vez, tienen una base genética (Bond, 2000; Fowler, 1995; Searight, 2000).

Los síntomas más comunes que se presentan son: distracción, impulsividad e hiperactividad. Es sumamente importante una evaluación profunda con terapeutas especialistas en el tema. La evaluación debe incluir un análisis exhaustivo de la historia familiar, médica y académica de las personas, como también todas las pruebas de habilidad y logros especializados para este diagnóstico (Bond, 2000; Fowler, 1995; McCormick, 1994).

Cada persona joven-adulta llega a niveles académicos, profesionales o personales en el transcurso de su vida, donde, si su problema o condición de ADHD no es atendida correctamente, puede llegar a sentir su autoestima baja e incluso, sentir una especie de frustración hacia sí (Bond, 2000; Nadeau, 1996).

A medida que pasan los años, son más las personas adultas diagnosticadas con esta condición, incluyendo aquellas que aún no se encuentran diagnosticadas. Además de que, con el paso del tiempo, muchas de estas diagnosticadas o no diagnosticadas pueden verse atraídas por adquirir una educación superior o universitaria.

Esto trae consigo un gran reto en la educación, ya que la gran mayoría de las personas con ADD tienden a mostrar conductas de inhibición, son inatentas y tienen muchas dificultades en mantener la atención en una tarea o actividad. Entre estos grupos existe quien puede poner atención a su tarea, pero pierde el enfoque por la posibilidad de hiperactividad e impulsividad (Asherson, Adamou y Bolea, 2010; Ortiz, et al., 1996).

Ello crea, en estos grupos, la dificultad de controlar sus impulsos y actividades, limitando las oportunidades de obtener su grado universitario, ya que pueden reflejar conductas inquietas o nerviosas al enfrentarse a actividades excesivas en los colegios o universidades (Quesada y Sáenz, 2009).

Por tanto, deben poner en práctica ciertas estrategias para el control de las aptitudes, que les faciliten el manejo de una conducta adecuada y que les ayuden a disminuir la conducta inadecuada en el salón de clases y en su ambiente. Deben recibir orientación y ayuda en cómo completar sus tareas educativas, sus actividades en los hogares, en las comunidades y hasta en sus trabajos, a fin de que puedan tener un mejor manejo y éxito de estos (McCormick, 1994; Latham y Latham, 1994; O’Connor, 1994).

Es de conocimiento que existen personas con ADHD o ADD y que pueden ser encontradas en las universidades. Sin embargo, muchos maestros y maestras de educación universitaria se encuentran con el creciente problema de conducta, en jóvenes de universidades o colegios. Esta conducta, que para el personal docente resulta inadecuada, puede crear cierto nivel de "estrés" (tensión agobiante) entre ambas partes. Esta fricción puede provocar el desinterés del estudiantado al curso tomado y puede crear bajas académicas (Lanberg y Epstein, 2008; Trampush, Miller, Newcorn y Halperin, 2009).

Con estas referencias, se debe investigar más a fondo el nivel de conocimiento del personal docente universitario. La falta de información o de conocimiento del ADHD pudiera estar impidiendo el debido reconocimiento y manejo del déficit de atención e hiperactividad en el estudiantado adulto universitario y se va haciendo difícil su detención.

No solo esta condición afecta el bienestar académico de este individuo, sino que remite contra todo lo que está a su alrededor. Estudios refieren que jóvenes con ADHD pueden involucrarse en problemas en sus trabajos, en sus hogares y hasta pueden abusar de sus medicamentos o drogas (Trampush, et al., 2009). 


\section{Marco teórico}

\section{Estudios generales}

En un estudio sobre las investigaciones contemporáneas realizadas por CHADD, Children and Adults with Attention Deficit/Hiperactivity Disorder demostró que de la niñez diagnosticada con ADHD, un 67\% continúa presentando los síntomas del trastorno en su edad adulta.

Estas manifestaciones interfieren significativamente con el funcionamiento académico, vocacional o social de su vida adulta. Estos datos destruyen la creencia en muchos profesionales de que niños, niñas y adolescentes superan sus síntomas de ADHD al llegar a la pubertad y seguramente al llegar a la adultez.

En adición, esta organización nos informa que los síntomas reflejados en la adultez comienzan a manifestarse en su niñez (alrededor de los 7 años) pero estos no fueron diagnosticados para ese entonces. Lo que provoca una identificación tardía en las personas adultas con ADHD.

Se indica que los síntomas en la edad adulta son llamados trastornos escondidos (síntomas asociados a otras condiciones). Estas señales se suelen esconder detrás de las relaciones personales, la organización, los trastornos del estado de ánimo, el abuso de sustancias, el empleo u otras dificultades psicológicas.

Es un trastorno complejo y difícil de evaluar. Solo debe ser diagnosticado por una persona profesional capacitada y con experiencia en el campo. Además, se puede reconocer el ADHD en el individuo adulto cuando este presenta problemas como la depresión, la ansiedad, el abuso de sustancias, el control de impulsos o cuando sus niños o niñas reciben este diagnóstico (Amen, 2001; Kessler, et al., 2006).

Según informes realizados por CHAD, esta condición afecta entre el 3 y el $5 \%$ de toda la niñez, quizás tanto como a 2 millones de estadounidenses. Los varones se ven afectados dos o tres veces más que las niñas. Al menos, un niño de cada aula escolar en los Estados Unidos necesita ayuda o tratamiento médico a fin de paliar los síntomas de este trastorno (CHAD The National Resource on ADHD, 2005). aunque se puede estimar que en los Estados Unidos se encuentra un 4.4\% de la población general (de 18 a 44 años) que está presentando la condición del déficit de atención e hiperactividad (Bauermeister, 2017, Kessler et al., 2006)

En el caso de Puerto Rico, Bauermeister (2017) indica que el 7.5\% de la población común de la niñez y la juventud puertorriqueñas entre las edades de 4 a 17 años presentan la condición del ADHD. Esta información fue revelada a raíz de una investigación que hizo Bauermeister en el 2007. En esta investigación, Bauermeister y su equipo entrevistaron varios grupos de padres y madres $(1,886)$ niños, niñas y adolescentes de pueblos rurales.

En la muestra utilizada, se encontró un grupo de participantes que recibía servicios mentales y de drogadicción por el sistema público en la isla. Mientras que en otra muestra se representó un grupo de la comunidad no asociados a los servicios sociales. Al comparar ambos participantes encontraron que los niños son más propensos a tener el ADHD que las niñas (CHAD, 2005). Aunque se reflejó que los síntomas del déficit de atención e hiperactividad se destacaron más en los grupos de niños y niñas que en los de adolecentes (Bauermeister, 2017).

De esta muestra se desprendió que el ADHD se manifiesta en grupos de niños y niñas con un historial médico, problemas educativos y de comportamiento. Además, se indicó que el déficit pudiera estar relacionado con aquellos tratamientos dados a los niños y niñas que nacieron prematuramente, a los problemas generales del habla y lenguage, a problemas en el dormir y a otros desórdenes encontrados en el Manual DSM-IV (2000).

Bauermeister (2017) indica que participantes que habían mostrado la condición deficitaria estaban con más propensión a recibir consejería, educación especial y podían tener un historial de suspenciones o expulsiones de las escuelas a las que asisten. Según los reportes tomados de las entrevistas hechas a las personas (padres, madres o familiares) que cuidan de los niños y niñas entrevistados, se encontró que habían sufrido en su niñez síntomas de hiperactividad o impulsividad. 
Incluso reportaron haber tenído enfermedades como la depresión, pérdida de trabajo, expulsiones o arrestos y haber estado sin ayudas sociales o económicas. Se refleja que estos grupos tienden a tener una baja calidad en la relación con el niño o la niña y proceden a hacer uso de diciplinas negativas con más amplitud en comparación con padres o familiares que no tienen un niño o niña con el déficit de atención e hiperactividad (Bauermeister et al., 2007). Se entiende que estos factores, además de la edad y el género pudieran estar específicamente relacionados con el ADHD en comparación con otros factores ya investigados.

No obstante, al comparar ambos grupos de participantes, Bauermeister (2017) encontró que los síntomas son más pronuciados en quienes participan del servicio de salud que en el grupo de niños y niñas de la comunidad que no había sido tratado por este programa. Las investigaciones (Bauermeister et al., 2007) refieren que para el momento del niño o niña llegar a la edad de 12 a 17 años, están más propensión a que se les refiera y trate por síntomas relacionados con desórdenes internos o conductas externas no relacionadas al ADHD. Se piensa que se debe estudiar más la relación que tiene la edad en los efectos del déficit de atención e hiperactividad y, a su vez, se recomienda que se lleven a cabo estudios longitudinales.

El déficit de atención e hiperactividad continúa manifestándose en algunas personas a lo largo de su vida, ya sea en la condición o en la manifestación de los síntomas. En ciertas investigaciones científicas se ha reportado que un 70 a $80 \%$ continúan presentando la condición en la adolescencia. Un 8 al $68 \%$ sigue presentando esta condición o al menos en un 50 a un 70\% los síntomas persisten sobre todo en aquellas personas que reflejan dificultades en sus funciones ejecutivas (Barkley, 2006; Barkley, Murphy y Fisher, 2008; Barkley, Murphy y Bauermeister, 1998; Bauermeister 2017).

Por otra parte, en una investigación longitudinal realizada a un grupo de primaria en Estados Unidos se comparó a los niños y niñas con déficit versus aquellos grupos de niños y niñas que no tienen la condición (Sheffler citado por Ramsey, 2009). A su vez, se comparó la relación de medicamentos con sus logros académicos. En ella se encontró que los niños y las niñas con ADHD tienden a tener bajos grados en matemáticas y en lectura. Además, son muestran más propensión a repetir grados o a salirse de la escuela. Sheffler (citado por Ramsey, 2009), de University of California Berkeley, y sus colegas analizaron una muestra de 594 niños y niñas con diagnóstico ADHD.

El grupo participante fue parte de un estudio de largo alcance mientras cursaba los grados escolares de primaria hasta el quinto grado. El estudio estaba enfocado en las asignaturas dirigidas a las matemáticas y a la lectura. Se pretendía corroborar si el uso de los medicamentos para tratar el ADHD estaba asociado a los logros y a los resultados académicos de estos grupos de estudiantes en la escuela elemental.

El estudio encontró que quienes tomaban medicamentos tenían una puntuación en matemática de 2.9 y en literatura reflejaban un 5.4 puntos más altos de quienes no tomaban medicamentos, pero que sí sufrían la enfermedad (Ramsey, 2009). Este progreso gradual se observó en las matemáticas durante el primer año de los 5 años estipulados del estudio. Mientras que en el área de la lectura se observaron los cambios en el primer tercio del año.

El estudiantado mejoró rápidamente en la lectura. Sin embargo, esto fue visto solo en aquel alumnado que había tomado medicamentos al menos por los primeros 2 años del estudio. Lo que puede indicar que una intervención temprana podria ayudar al individuo con ADHD en su cognición.

Luego, en otro estudio realizado por Biederman, Petty, Ball y Doyle (2009) se tomaron el tiempo de analizar una muestra de 217 sujetos entre las edades de 15-31 años con ADHD o sin este, quienes fueron evaluados en 3 periodos durante 10 años en su adolescencia y adultez.

Los sujetos presentaban la condición de ADHD entre moderado y persistente. Los grupos con ADHD se compararon con aquellos que no poseían la condición y se encontró que tenían puntuaciones bajas en todo lo relacionado a la cognición. Esta deficiencia cognitiva es relativamente estable en personas jóvenes adultas y parece ser independiente en el curso del ADHD.

Estos datos y otras investigaciones apuntan a que el $\mathrm{ADHD}$ se asocia con los bajos grados, problemas con la lectura y las matemáticas, bajas puntuaciones en los exámenes estándares y que tienen relación con un gran 
incremento en la retención de grados (repetición de grados). Además, esta condición se asocia con un alto uso de servicios escolares, aumento de detenciones y expulsiones, y últimamente, a un relativo bajo nivel de estudiantado graduando en la escuela superior (Fletcher y Wolfwe, 2008; Nadeau, 1996).

\section{Estudios a docentes}

No hay muchos estudios enfocados en docentes que imparten clases en las universidades. Sin embargo, se encontró este estudio enfocado en la opinión o percepción que tenían los maestros y maestras cuando trabajaban con adolescentes con diagnóstico de déficit de atención e hiperactividad.

Rush y Harrison (2008), ambos de la universidad de Alabama, usaron la técnica de "conceptos mentales" donde examinaron la percepción de los maestros sobre los adolescentes con ADHD. Los investigadores entendían que era preciso conocer la percepción de los maestros ya que es importante que los profesores consideren realizar planeaciones y que estos creen estrategias para ayudar al estudiante académicamente.

En esta investigación participaron 100 docentes de educación general. Estos fueron escogidos de cuatro escuelas superiores en la Florida. Se les entregó un paquete de tarjetas que incluían datos o temas perceptuales. Debían dividir cada tema y combinarlo con los datos que aplicara, según su percepción. La clasificación de los temas fue evaluada a través de escalas o valoraciones numéricas y mapas grupales.

Se seleccionaron cinco temas principales sobre la percepción docente. El primer tema estaba relacionado con la confidencia y los retos al enseñar a esta comunidad. Se indicaron temas o percepciones como deseo que el/la adolescente se sienta más aceptado/a y cada adolescente es diferente (Rush y Harrison, 2008).

El segundo tema estaba relacionado a la frustración y dificultades de los maestros y maestras al educarles. Se indicó temas como: me siento incómodo/a enseñando a adolescentes con ADHD y me siento frustrado/ a con los padres de adolescentes con ADHD (Rush y Harrison, 2008).

En el tercer tema se relacionó con los problemas de comportamiento donde se incluyó: el comportamiento de un/a adolescente con ADHD es siempre disruptivo y "los/las adolescentes con ADHD usan la condición como una excusa (Rush y Harrison, 2008).

El cuarto tema estuvo relacionado con problemas en el salón de clases (ayudas) indicando que:no tengo mucho material para ayudar a adolescentes con ADHD y yo cambio el currículo a causa de adolescentes con ADHD (Rush y Harrison, 2008)

Por último, el grupo cinco fue relacionado con la necesidad de un entrenamiento sobre el déficit del ADHD, el uso de la disciplina y el manejo del salón de clases, donde indicaba que: necesito más entrenamiento, específicamente en adolescentes con ADHD y adolescentes con ADHD no deben estar en clases grandes (Rush y Harrison, 2008).

Este último tema fue clasificado en el área central del mapa conceptual. Lo que indica que su percepción gira en torno a la necesidad de tener o adquirir conocimiento sobre las técnicas de disciplina y un buen manejo de clase.

Otra interpretación es que docentes con una percepción negativa sobre el trabajar con adolescentes con ADHD pueden sentir incomodidad o encontrar que es difícil tener a estos grupos de estudiantes en clase, debido a que no han recibido un entrenamiento adecuado sobre el ADHD, que les prepare para una adecuada atención.

También se observó que los maestros y maestras que tienen una percepción positiva al trabajar con adolescentes con ADHD pueden conceptualizar que el educar a esta población puede ser más productivo si ellos o ellas tienen más entrenamientos en ADHD.

En resumen, su percepción está enfocada en expandir los conocimientos generales sobre el ADHD y aumentar las destrezas en el uso de estrategias que intervengan con el estudiantado adolescente y su condición. Si el personal docente es educado y entrenado para trabajar con esta comunidad deficitaria, el alumnado tendrá más posibilidades de alcanzar sus metas. 
Siguiendo la línea investigativa en el personal docente, este estudio realizado por Jarque y Tárraga (2009) comparó el conocimiento que tiene el activo y el futuro sobre el trastorno por déficit de atención con hiperactividad (TDAH) y sus características.

En el estudio que realizaron Jarque y Tárraga (2009) participaron tres grupos en total. El primer grupo compuesto por 265 estudiantes de 1 ro de Magisterio de la Escuela Universitaria de Magisterio. La muestra estaba compuesta por 208 mujeres y 48 varones; 19 sujetos no especificaron su género.

La edad media de los sujetos participantes era de 21 años, sin incluir 5 sujetos que no aportaron esta información. El 45.5\% indicó que había recibido formación específica en TDAH durante una media de tres horas. Mientras que un $6.9 \%$ tenía experiencia docente con niñas o niños hiperactivos durante una media de 0.57 meses.

El segundo grupo estaba formado por 130 estudiantes de 3ro de Magisterio de la Escuela Universitaria de Magisterio. Había 120 mujeres y 10 varones que participaron en la investigación. La edad media era de 28 años. Un 89\% había recibido formación del TDAH por un tiempo medio de 9.5 horas. El 32.3\% tenía experiencia docente con estudiantes con TDAH, durante una media de 1.39 meses.

En el tercer grupo conformado por 193 docentes activos, de cuyo grupo 67 impartían docencia en la etapa de Educación Infantil y 125 en la etapa de Educación Primaria. La muestra estuvo compuesta por 130 mujeres y 43 hombres. La edad media de los sujetos era de 42 años (Jarque y Tárraga, 2009).

Tenían una media de 17 años de enseñanza docente y más de la mitad había tenido alguna experiencia docente con niñez con TDAH, durante una media de 14 meses. Además, un 51.6\% había recibido formación sobre el TDAH, durante una media de 7.80 horas.

Se le administró un cuestionario que estaba agrupado por temas específicos. Se le llama el KADDS (Knowledge of Attention Deficit Hyperactivity Disorder) adaptado al español, compuesto por 36 preguntas de tres alternativas de respuesta (verdadero, falso, no sé).

Estas preguntas estaban agrupadas por categorías: información general sobre la naturaleza, causa y repercusiones del TDAH (15 preguntas); síntomas/diagnóstico de TDAH (9 preguntas) y tratamiento del TDAH (12 preguntas). Se utilizó este sistema ya que ha mostrado ser eficaz y fiable según indican las investigaciones (Jarque y Tárraga, 2009).

Para evaluar la autoeficacia percibida se añadió una pregunta que decía: “¿Hasta qué punto consideras que puedes enseñar de forma efectiva a un/a niño/a con TDAH?” Esta pregunta debía ser contestada utilizando una escala de valor de 7 puntos, donde 1 era el extremo inferior (personal no preparado para enseñar de forma efectiva a un niño o niña con TDAH) y 7 era el extremo superior (personal totalmente preparado).

De la data se desprende que, al analizar las tres muestras de sujetos, estas indicaron que el nivel de conocimiento sobre el TDAH es bastante escaso, ya que han contestado adecuadamente a menos de la mitad de las preguntas del cuestionario. Los hallazgos son sensiblemente inferiores a datos encontrados en otros estudios previos que, también han empleado muestras de estudiantes de magisterio y personal docente activo.

De estos hallazgos se deriva una reflexión sobre la necesidad de ampliar la formación continuada que recibe el personal docente a lo largo de su carrera, sobre aquellos trastornos, con tanta incidencia como el TDAH (Jarque y Tárraga, 2009).

Jarque y Tárraga (2009) indican que el personal docente activo y en formación está en la necesidad de recibir una formación teórico-práctico en el manejo del TDAH en la escuela. Mejorar sus relaciones con la confianza en sí, para dar respuestas educativas adecuadas a este tipo de estudiantes. Se debe, por tanto, continuar más investigaciones que ayuden a entender el porqué de las limitaciones, para recibir entrenamiento sobre el déficit a todo nivel escolar.

En otro estudio realizado por Martinussen, Tannock y Chaban (2011), investigaron a docentes de educación especial y educación regular (general) sobre el nivel de entrenamiento que tenían del déficit de atención e hiperactividad (ADHD) y la relación entre el nivel de entrenamiento en el ADHD y el 
uso instruccional recomendado al enfocar el manejo del comportamiento en estudiantes que exhiben un problema de conducta.

El análisis reveló que la mayoría de docentes regulares (76\%) y casi la mitad (41\%) de educación especial, reportan no haber tenido ningún o muy poco entrenamiento/desarrollo profesional sobre el ADHD.

El profesorado regular que cuenta con un moderado o mayor número de entrenamientos extensivos sobre el ADHD reporta un marcado aumento en el uso de recomendaciones enfocadas al manejo del comportamiento, que el regular con poco o ningún entrenamiento sobre el ADHD.

Martinussen, Tannock y Chaban (2011) indican que recientes estudios han demostrado que los niños o niñas que exhiben problemas de comportamiento en la clase, particularmente quienes muestran inatención o hiperactividad, están a riesgo de tener un bajo rendimiento académico y de salirse de la escuela.

Se sugiere que el personal docente debe tener un buen entendimiento del tipo de instrucción por utilizar, que esté enfocada en el manejo del comportamiento, y que, efectivamente, promueva un compromiso y un progreso académico en el estudiantado.

Sin embargo, algunos datos tienden a puntualizar que los maestros y maestras pueden sentir que no tienen preparación para enseñar a niñez con problemas de atención o de comportamiento, limitando el éxito académico del estudiantado.

Se entiende que mucho de este estudiantado con ADHD es mayormente enseñado en una clase regular por docentes regulares. Se ha encontrado que gran cantidad de docentes regulares está falta de entrenamiento sobre la condición del ADHD. En un estudio realizado en América y Canadá, se encontró que los maestros o las maestras practicantes no reciben entrenamiento sobre la condición del ADHD durante su programa de preparación.

Otro reporte informó que la mayoría (77\%) de una muestra de practicantes en Australia no recibió un preentrenamiento sobre la condición del ADHD. Bekle (2004) encontró que muy poco personal practicante recibió un "comprensivo" entrenamiento (oportunidad de desarrollo profesional) que abarcara el tema del ADHD. Esta falta de entrenamiento puede afectar el progreso académico del estudiantado.

Martinussen, Tannock y Chaban (2011) refieren que hay una preocupación sobre la educación, ya que docentes regulares con muy poco entrenamiento en la condición del ADHD pueden tener un bajo nivel de conocimiento sobre esta condición y poca actitud positiva hacia estudiantes con ADHD (Bekle, 2004).

Los resultados obtenidos de esta investigacion sugieren que, si se les provee entrenamiento a los maestros y maestras generales de cómo trabajar efectivamente con estudiantes con ADHD, puede aumentar el uso de las instrucciones recomendadas, enfocadas en el manejo del comportamiento y el manejo de estrategias de las ayudas instruccionales (Zental y Javorsky, 2007, citado por Martinusen et al., 2011).

Por lo tanto, participar en un entrenamiento sobre estudiantes con problemas de conducta debe ser facilitado a ambos tipos de profesorado, novato y experimentado. Estos entrenamientos pueden ayudarles a trabajar, en clase, más efectivamente con estudiantes que exhiben problemas en su comportamiento. Ayudará a fortalecer su conocimiento y asegurará el éxito académico del estudiantado.

Se deben hacer más investigaciones que examinen la forma en la que mejor se pueda expandir el conocimiento de los maestros y las maestras regulares y de educación especial, sobre el ADHD y el uso de más instrucción individualizada que ayude y enfoque el manejo del comportamiento para facilitar el éxito académico de estudiantes con problemas de conducta.

\section{Objetivos generales (Tabla 1)}

- Conocer las opiniones o el conocimiento del profesorado acerca del TDAH (ADHD siglas en inglés) en las personas jóvenes adultas.

- Recopilar datos sobre los diferentes métodos que utilizan para impartir la enseñanza a jóvenes de universidad con esta condición. 
- Conocer las opiniones del profesorado sobre el trato, acomodo y apoyo ofrecidos en las universidades para estudiantes con TDAH (ADHD).

TABLA 1

Objetivos e indicadores

\begin{tabular}{|c|c|}
\hline Objetivos & Indicadores de evaluación \\
\hline $\begin{array}{l}\text { Conocer las opiniones o el conocimiento } \\
\text { del profesorado acerca del TDAH (ADHD } \\
\text { siglas en inglés) en las personas jóvenes } \\
\text { adultas. }\end{array}$ & $\begin{array}{l}\text { Se realizó un cuestionario donde se les } \\
\text { preguntaba cuánto conocian sobre el } \\
\text { tema del ADHD. Si habian escuchado } \\
\text { sobre esta condición en las personas } \\
\text { adultas. }\end{array}$ \\
\hline $\begin{array}{l}\text { Recopilar datos sobre los diferentes } \\
\text { métodos que utilizan para impartir la } \\
\text { enseñanza a jóvenes de universidad. }\end{array}$ & $\begin{array}{l}\text { Se hicieron preguntas abiertas en el } \\
\text { cuestionario sobre los métodos de } \\
\text { enseñanza que utilizaban en sus salones } \\
\text { de clases. Se les preguntó si hacian } \\
\text { cambios en el uso de métodos. }\end{array}$ \\
\hline $\begin{array}{l}\text { Conocer las opiniones del profesorado } \\
\text { sobre el trato, acomodo y apoyo que son } \\
\text { ofrecidos en las universidades. }\end{array}$ & $\begin{array}{l}\text { Esta información fue recopilada a través } \\
\text { del cuestionario administrado al } \\
\text { profesorado. Se le preguntó su opinión } \\
\text { al respecto. }\end{array}$ \\
\hline
\end{tabular}

\section{MÉTodo}

\section{Sujetos}

Para realizar esta investigación, se contó con la colaboración de varios colegios y universidades en las zonas rurales y metropolitanas del país. En estos centros educativos se obtuvo la participacion de 100 docentes que impartían clases en diferentes facultades. Las universidades y colegios participantes fueron: la Universidad Metropolitana, la Universidad del Este, la Universidad de Cabo Rojo y el Colegio Columbia College. Se utilizó un cuestionario con 27 preguntas abiertas y semiabiertas formuladas con base en los objetivos establecidos para esta investigación (Anexo 1).

Las edades del cuerpo docente fluctuaban entre los 25 y 55 años. Este está compuesto de 48 hombres y 52 mujeres. Su nivel socioeconómico al momento de la investigación era de nivel medio a un nivel alto. Además, se encontró que un 35\% contaba con 1 a 5 años de experiencia, 22\% tenía unos 6 a 10 años de experiencia y un $43 \%$ tenía más de 10 años de experiencia en el ejercicio docente.

\section{Diseño y procedimiento}

En este estudio se prefirió utilizar un método cualitativo a fin de rocoger ciertos datos y opiniones del profesorado entrevistado. Para obtener dicha información, se elaboró una entrevista que constaba con preguntas abiertas y cerradas. De acuerdo con los parámetros de una entrevista, esta es considerada como una técnica que provee información profunda sobre un problema en particular. En este caso, teníamos la necesidad de conocer cuánto sabían el profesorado sobre el tema del déficit de atención e hiperactividad en la persona adulta.

Como ya establecimos, una de las modalidades utilizadas en el cuestionario fue el uso de preguntas abiertas o semiabiertas, lo que se asemeja más a una conversación natural, donde se obtiene una información valiosa, sin tener que recurrir a un estilo de interrogación directa, por lo que se elaboró un cuestionario de 27 preguntas relacionadas con los objetivos trazados para dicha investigación. Este medio de recogida de 
información permitió ampliar los datos obtenidos y le ofreció al sujeto entrevistado la flexibilidad necesaria para formular sus respuestas y la oportunidad de aclarar sus propias declaraciones.

Se señaló un día entre semana, para hacerles llegar los cuestionarios al profesorado del colegio técnico y otro día diferente para hacer entrega en las universidades. El cuestionario contaba con 27 preguntas. Luego de contestarlo, se hacía una entrevista personal para aclarar o expandir algunas de sus respuestas.

La entrevistadora le indicó al profesorado el propósito principal (saber acerca del conocimiento de los maestros y las maestras sobre las personas adultas con ADHD en los centros de estudios) de la investigación sin informar sobre los objetivos planteados en el estudio.

Además, se entregó un cuestionario donde debían llenar sus datos sociológicos, el cual incluía datos comunes como nombre, apellido, edad, entre otros. También contenía preguntas relacionadas con las nacionalidades de sus padres y madres, tiempo ejercido como docentes y el lugar de estudios superiores.

Ambos días, se le pidió al profesorado que, en confidencialidad, depositara en el cuestionario todo el conocimiento que tenía con relación al tema de las personas adultas con déficit de atención e hiperactividad. La entrevista tuvo una duración de 45-60 minutos, incluido el tiempo de entrevista personal a fin de aclarar algunas respuestas.

Mientras contestaban las preguntas, también se indicó la existencia del cuestionario específico sobre el ADHD del manual de diagnóstico DSM-IV para identificar los posibles individuos con ADHD. Esto se hizo con el propósito de que, eventualmente, busquen este formulario y se lo administren a sus estudiantes.

\section{Variable contemplada}

Debido a la amplia muestra de profesorado entrevistado, se señaló como una variante los años de experiencia que este tenía en el campo educativo. Frecuentemente, se tiende a creer que una persona que lleve más años en un campo profesional específico es una persona experimentada versus aquel profesional que solo cuenta con varios años de trabajo en su campo profesional, en este caso la educación. Se deseaba saber si los años de trabajo como educativo intervendrían en la capacidad docente para reconocer el déficit en el estudianado, ya que se ha observado que la "experiencia" no define el conocimiento docente sobre estudiantes con ADHD (Jarque y Tárraga 2009). Se estableció que los años de experiencia no afectan o intervienen en la capacidad que pueda tener un maestro o una maestra sobre la condición deficitaria. En otras palabras, la experiencia no determina el conocimiento del profesorado sobre el tema.

En la Tabla 2 "Años de experiencia" se muestra el tiempo dedicado a la educación de las maestras y los maestros entrevistados.

TABLA 2

Años de experiencia

\begin{tabular}{lc}
\hline $\begin{array}{l}1 \text { a } 5 \text { años de experiencia } \\
\text { profesores/as }\end{array}$ & 35 \\
\hline 6 a 10 años de experiencia & 22 \\
profesores/as & \\
\hline $\begin{array}{l}10 \text { o más años de experiencia } \\
\text { profesores/as }\end{array}$ & 43 \\
\hline
\end{tabular}

\section{Resultados}

Al realizar la entrevista, una de nuestras preguntas estaba enfocada a evaluar el conocimiento del profesorado sobre el TDAH (ADHD) en las personas jóvenes adultas. Se les preguntó; ¿Ha escuchado hablar sobre el 
déficit de atención e hiperactividad (ADHD) en personas jóvenes adultas? ¿Como qué? ¿Cuánto conoce del tema?

Se recopilaron respuestas tales como; "si, conozco algo, un poco, bastante", "no, conozco muy poco, necesito más información", "niños que nacen prematuramente, tienen problema de aprendizaje, se distraen, necesitan medicamentos, falta de comunicación, dificultad para expresarse..." (Tabla 3).

TABLA 3

Conocimiento del ADHD

\begin{tabular}{|c|c|c|}
\hline $\begin{array}{l}\text { \#Personal } \\
\text { docente }\end{array}$ & $\begin{array}{c}\text { Conocimiento } \\
\text { del ADHD }\end{array}$ & $\begin{array}{c}\text { Resultados } \\
\mathbf{2 0 1 0}\end{array}$ \\
\hline \multirow{8}{*}{100} & a & $>20$ \\
\hline & b & 15 \\
\hline & $\mathrm{c}$ & 10 \\
\hline & d & 15 \\
\hline & $\mathrm{e}$ & 12 \\
\hline & $\mathrm{f}$ & 10 \\
\hline & $\mathrm{g}$ & 10 \\
\hline & $\mathrm{h}$ & 8 \\
\hline
\end{tabular}

\footnotetext{
Nota: a.si, el nacimiento prematuro desarrolla esta condición. Condición que no ayuda en la concentración. Interrumpen clases y hablan mucho. b. si, solo he escuchado el tema en niños, se de algunos adultos con esta condición, actualmente se habla de la condición en adultos. c.si, llaman la atención de todos alrededor, intranquilos, se distraen con facilidad, la mayoría son competentes e inteligentes. d. si, se vuelven agresivos y necesitan medicamentos, problemas de aprendizaje, problemas de aprendizaje específicos. e. si, después de adultos muchos son diagnosticados, no desean tomar medicamentos, hacen tareas que para ellos son agotadoras. f. si, a través de medios de comunicación, falta de habilidades como la concentración, comunicación y formas de expresarse. g. no, muy poca información, e desea saber más sobre el tema. h. no, n/a
}

Con base en los datos encontrados, se observó que una gran parte del profesorado (más del 60\%) tiene conocimiento del déficit de atención e hiperactividad en el individuo. Este conocimiento está basado en la información suministrada sobre los niños y las niñas con $\mathrm{ADD}$ o ADHD. De la muestra que aquí referimos, se desprendió que un $45 \%$ reconoce la condición en la niñez y alrededor de un $20 \%$ refiere conocer sobre la condición en la adultez.

De acuerdo con la entrevista personal, este conocimiento está basado en lo que han escuchado, visto o les han dicho a lo largo del tiempo o de ejercer sus carreras educativas. Este detalle nos indica que aún ignoran el hecho de que este problema persiste a lo largo de la vida (Colleen y Elliot, 1995) no importando sus años de experiencia en el campo educativo.

Cabe señalar que los sujetos entrevistados contribuyeron con sus opiniones e indicaron la falta de información que se tiene sobre el ADHD. Refieren conocer sobre los problemas de aprendizaje e incapacidades mentales; pero no tienen la información necesaria que les indique sobre la perseverancia de los síntomas en las personas adultas con ADHD y sobre el trato educativo para esta comunidad (Martinussen, Tannock y Chaban, 2011). Por tanto, la variable contemplada sobre los años de experiencia no produjo mayor efecto sobre su conocimiento acerca del déficit de atención e hiperactividad.

Otras de las preguntas estuvieron enfocadas en los métodos que el profesorado utilizaba al reconocer la condición en sus estudiantes. La pregunta fue la siguiente: ¿Hace usted algunos cambios en su método de enseñanza-aprendizaje cuando identifica algún problema de aprendizaje o conducta en sus estudiantes? 
Algunas de las respuestas recopiladas fueron las siguientes; "si, utilizo métodos variados y llamativos que ayuden a captar la atención del alumno, adaptar la enseñanza, uso de horas extras", "si, asistencia individualizada, dibujos, juegos explicativos, uso de apuntes, dictado, lápizy papel", "realizo cambios educativos, aunque no tenga el debido adiestramiento..."(Tabla 4)

TABLA 4

Métodos de aprendizaje

\begin{tabular}{|c|c|c|}
\hline $\begin{array}{c}\text { \# Personal } \\
\text { docente }\end{array}$ & $\begin{array}{l}\text { Métodos de } \\
\text { aprendizaje }\end{array}$ & $\begin{array}{c}\text { Resultados } \\
2010\end{array}$ \\
\hline \multirow{7}{*}{100} & a & $\rightarrow \quad 21$ \\
\hline & $\mathrm{b}$ & 11 \\
\hline & $\mathrm{c}$ & 15 \\
\hline & $\mathrm{d}$ & 25 \\
\hline & e & 11 \\
\hline & $\mathrm{f}$ & 12 \\
\hline & $\mathrm{g}$ & 5 \\
\hline
\end{tabular}

\footnotetext{
Nota: a. si, utilizo métodos variados, llamativos que me ayudan a captar su atención por periodos más largos, dar horas extras. b. si, adapto la enseñanza, creo actividades para aquellos estudiantes que son más lentos, adapto según habilidades y necesidades, uso de demostraciones. c. si, trabajar más cerca del estudiante, uso de estudiantes como recurso en clase, estudiante trabaje con otro estudiante. d. si, educación, asistencia individualizada, dinámica en grupos, capten atención e interés del estudiante, uso de preguntas para comprobar lo aprendido, uso de apuntes, dictado, uso de papel y lápiz. e. dibujos, juegos explicativos, traducción de términos usados en clase, uso de audiovisuales, explicar materiales educativos. f. combinar enseñanza con métodos visuales y auditivos/computadoras, hacer cambios, aunque no tenga el debido adiestramiento, seguimiento de guía educativa/currículo. g. n/a
}

Al preguntarle al profesorado acerca de su pericia a la hora de identificar alguna condición en sus salones de clases observamos que cada docente hace lo pertinente para captar la atención de su estudiantado. Alrededor de un $60 \%$ a un $80 \%$ de docentes indica que utiliza diferentes métodos educativos que le ayudan a atender las necesidades de sus estudiantes. Trabaja con el estudiantado a fin de adaptar las clases a sus habilidades. Realiza trabajos en grupos, da horas extras para realizar trabajos, entre otros (Nadeau, 1996; Zeigler, 1995).

Cuando el maestro o la maestra puede identificar a este alumnado, refieren que deben recurrir a libros, internet y otros recursos para complementar sus clases/currículo y cubrir sus necesidades educativas, lo que indica que el centro académico no está proveyendo la información o herramientas necesarias para trabajar con estos individuos (Rush y Harrison, 2008).

Otro grupo de docentes refiere que, de tener algún o alguna estudiante con dificultades académicas, consulta al profesorado más experimentado, a fin de conocer y poner en práctica algún método factible y apropiado. Además, indica que recurren a clases continuas para mejorar sus conocimientos y poder así trabajar con esta población (Martinussen, Tannock y Chaban, 2012).

Se le hizo una pregunta al profesorado en referencia a su opinión sobre el trato educativo de este estudiantado. La pregunta fue la siguiente; ¿Cuál es su opinión en relación con el trato educativo que reciben jóvenes con $\mathrm{ADHD}$ ?

Las respuestas fueron variadas y en la mayoría de los casos, negativas. Estas fueron algunas respuestas; "es pobre, el estudiante está pasando desapercibido, caen en manos de maestros inadecuados, suelen desertar o fracasar..." (33\%), "creo que se les trata bien, depende del maestro, los profesores necesitan educarse, no todos sabemos lidiar con el problema..." (10\%), "depende, hay ayudas para estos estudiantes si se conoce la 
situación..." (10\%), "no, se les trata a todos por igual..." (25\%), “no, deberian recibir acomodos, los profesores deben estar capacitados para ayudarles...” (17\%), “no sé, n/a, no...” (5\%).

La mayoría del personal docente entiende que el trato y el acomodo académico no es el "ideal" para ayudar a estos grupos de jóvenes con discapacidades. La falta de información o desarrollo profesional esta impidiendo un mejor reconocimiento del ADHD y, por consiguiente, le está impidiendo al estudiantado el obtener mejores resultados académicos (Jarque y Tárraga, 2009).

\section{Discusión O CONCLUSIONES}

Del estudio se desprende que el profesorado no tiene una basta información sobre los individuos adultos con ADHD. Este resultado se ha visto en otras investigaciones realizadas a docentes en otras áreas educativas (Jarque y Tárraga, 2009; Martinussen, et al., 2011). La falta de información o de talleres educativos sobre el tema puede crear barreras en el aprendizaje del individuo.

Aunque el cuerpo docente reconoce la necesidad de capacitarse sobre el tema, el ADHD sigue siendo la causa en los problemas de cognición y de bajos grados académicos obtenidos por estudiantes (Fletcher y Wolfwe, 2008). Por tanto, es imprescindible que el profesorado sea capacitado y entienda las repercusiones académicas del deficit de atención e hiperactividad en personas jóvenes adultas.

Una detección temprana del déficit y su pronta atención podrían ayudar a la persona en el manejo de su condición. Esto le ayudaría en su organización y en su capacidad para desarrollar favorablemente su cognición (McCormick, 1994). Es necesario entender que el estudiantado joven está expuesto a un incremento de los síntomas deficitarios con el paso de los años. Por lo que una intervención en la niñez podría controlar los síntomas y el individuo podría tener mejor calidad de vida.

Por otra parte, en la investigación se observó la disposición del profesorado por utilizar métodos de enseñanza-aprendizaje que pudieran ayudar a estudiantes con su discapacidad y en su adquisición del conocimiento (McCormick, 1994; Latham y Latham, 1994; O’Connor, 1994). Los métodos utilizados pueden ser de gran ayuda cuando se implementan adecuadamente, ya que el "método" puede ser efectivo si es acompañado de los objetivos que se quieren alcanzar con el estudiantado.

Se entiende que, aunque el profesorado no había tenido el debido adiestramiento sobre el tema, estaba dispuesto a atender las necesidades estudiantiles (de tener algún caso en su clase) y tenía el compromiso de hacer llegar la educación de una manera u otra. Aquí se vio la necesidad de evaluar al estudiantado, para lo cual se utilizó un cuestionario usado para detectar sintomas asociados al déficit.

Este hecho fue una de las limitaciones del estudio, ya que la universidad no permitió la administración de este cuestionario a estudiantes. De esta forma, se limitó la corroboración (a través del cuestionario DSM-IV) de la existencia de estos síntomas en el alumnado.

Por otra parte, Rush y Harrison (2008) concuerdan en que el personal docente debe expandir los conocimientos generales sobre el ADHD y debe aumentar las destrezas en el uso de estrategias que intervengan con adolescentes y su condición. Según indican, si el profesorado recibe educación y entrenamiento para trabajar con esta comunidad deficitaria, el alumnado tendrá más posibilidades de alcanzar sus metas.

Otro aspecto que no se puede ignorar es el trato y acomodo que se les da a este estudiantado en el colegio. La mayoría de docentes (alrededor de 80\%) en nuestro estudio refiere que no se está haciendo lo necesario para ayudar a jóvenes con problemas deficitarios (entiendase el ADHD). El "déficit” no está siendo debidamente reconocido en las universidades y el estudiantado no esta recibiendo las ayudas necesarias que requiere su condición.

En resumen y de cara al futuro, se necesita una pronta atención y orientación de los cuerpos docentes y centros educativos hacia esta comunidad, ya que su falta de información y de reconocimiento pueden estar generando los bajos números de retención en las universidades. La incapacidad de maestros, maestras 
e instituciones en reconocer y tratar, apropiadamente, el ADHD puede estar afectando el éxito académico del individuo a nivel universitario.

Se recomienda hacer más estudios que permitan recolectar información sobre el tema. Esto nos ayudará a tener un mejor entendimiento sobre cómo esta condición pudiera estar afectando el rendimiento académico del estudiantado universitario.

Se puede sugerir que dichos estudios estén relacionacionados con investigaciones que identifiquen las nuevas alternativas y estrategias educativas aplicadas en las universidades, que estén ayudando al sistema cognoscitivo del estudiantado universitario. También se podría corroborar la existencia de recursos profesionales que sean necesarios para trabajar y educar a las personas con déficit.

Se debiera, a su vez, investigar y evaluar las alternativas académicas vigentes que permitan un cambio en el sistema educativo. Evaluar cuáles son aquellas limitaciones en los centros académicos que están impidiendo un acomodo fiable para esta comunidad estudiantil o los aspectos que contribuyen a la falta de orientación del personal docente activo, entre otros.

\section{REFERENCIAS}

Amen, D. G. (2001). Healing ADD The Breakthrough Program that Allows you to See and Heal the 6 Types of ADD [Sanando el déficit de atención. Un progreso en el programa que permite ver y sanar los seis géneros del déficit de atención]. New York, NY: Pinguin Group.

Asherson, P., Adamou, M. and Bolea, B. (2010). Is ADHD a valid diagnosis in Adult? Yes [¿Es el ADHD un diagnóstico válido en Adulto? Sí]. doi: https://doi.org/10.1136/bmj.c549

Barkley, R. A. (2006). Attention-deficit hyperactivity disorder: A handbook for diagnosis and treatment [ Trastorno de déficit de atención con hiperactividad: un manual para el diagnóstico y el tratamiento]. (3era ed.). New York: Guilford Press.

Barkley, R., Murphy, K. y Bauermeinster, J. (1998). Trastorno por déficit de atención e hiperactividad: Un manual de trabajo clínico. New York: Guilford Press.

Barkley, R. A., Murphy, K. R. y Fischer, M. (2008). ADHD in adults. What the science says [TDAH en adultos. Lo que dice la ciencia]. New York: Guilford.

Bauermeister J. J. (2017). El trastorno del déficit de atención e hiperactividad (TDAH) Recuperado de http:// atenciondrbauer.com/el-tdah-en-puerto-rico/

Bauermeister, J. J., Shrout, P. E., Ramírez, R., Bravo, M., Alegría, M., Martínez-Taboas, A., ... Canino, G. (2007). ADHD Correlates, Comorbidity, and Impairment in Community and Treated Samples of Children and Adolescents [Déficit de atención e hiperactividad, correlaciones, comorbilidad y discapacidad en comunidades y una muesta atendida/medicada de niños y adolescentes]. J Abnorm Child Psychol, 35(6), 883-898. Doi: $10.1007 / \mathrm{s} 10802-007-9141-4$

Bekle, B. (2004). Knowledge and attitudes about attention-deficit hyperactivity disorder (ADHD): A comparison between practicing teachers and undergraduate education students [Conocimientos y actitudes sobre el trastorno por déficit de atención / hiperactividad (TDAH): una comparación entre los profesores en ejercicio y los estudiantes de educación de pregrado]. J Atten Disord, 7(3), 151-61. Doi: 10.1177/108705470400700303

Biedermand, J., Petty, C. R., Ball, S. W. y Doyle, A. E. (2009). Are Cognitive Deficits in ADHD related to the course of the Disorder? A prospective controlled Follow-up Study of Grown up boys with Persistent and Remitting Course [¿Está el déficit cognitivo asociado al trayecto del desorden? Un estudio longitudinal en niños que han crecido con un trayecto persistente y remitido]. Journal of Psychiatry, 170(2-3), 177-182.

Bond, K. (2000). Adult ADHD, Could you have this disorder? [Adultos con déficit de atención e hiperactividad: ¿Podría tener este déficit?]. Recuperado de http://www.todayschristianwoman.com/ articles/2000/november/18.74.html

CHAD The National Resource on ADHD. (2005). Children and Adults with Attention Deficit/Hyperactivity Disorder [Niños y adultos con déficit de atención e hiperactividad]. Recuperado de http://www.chadd.org 
Colleen, A. R. y Elliot, P. T. (1995). ADHD \& Teens A parent's Guide to Making it Through the Tough Years [Déficit de atención e hiperactividad y adolescentes. Una guia a padres para que pasen los años difíciles]. Lanham MD: Rowman \& Littlefield, Publishing Group.

Diagnostic and Statistical Manual of Mental Disorders. DSM-IV. (2000). Manual de diagnóstico y estadístico de los trastornos mentales. American Psychiatric Association. USA: APA. Publisher in United States of America.

Fletcher, J. y Wolfe, B. (2008). Child mental health and human capital accumulation: the case of ADHD revisited[ La salud mental infantil y la acumulación de capital humano: el caso del TDAH revisitado]. J Health Econ. 27(3): 794-800. doi: 10.1016/j.jhealeco.2007.10.010

Fowler, M. (1995). Desorden déficitario de la atención [Trad. B. McCormick]. Recuperado de http:// www.ldonline.org/article/5788/

Jarque, S. y Tárraga, R. (2009). Comparación de los conocimientos sobre el trastorno por déficit de atención con hiperactividad (TDAH) de los maestros en activo y los futuros educadores. Fundación Infancia y Aprendizaje, 32 (4), 517-529.

Kessler, R.C., Adler, L., Barkley, R., Biederman, J., Conners, C., Demler, O.... Zaslavsky, A. (2006). The Prevalence and Correlates of Adult ADHD in United States. Results from the National Comorbidity Survey Replication [La prevalencia y correlaciones del adulto con déficit de atención e hiperactividad en la Unión Europea. Resultados en la réplica de encuestas en la comorbilidad nacional]. Am J Psychiatry, 163(4), 716-723. Doi: 10.1176/ appi.ajp.163.4.716

Lanberg, J. M. y Epstein, J. (2008). Make the Transition from Elementary School to Middle School Difficult for Kids with $\mathrm{ADD}$ [Haciendo la transición de escuela elemental a la intermedia difícil para niños con déficit de atención]. Journal of Child and Adolescent Psychology, 15(2), 113-121.

Latham, P. y Latham, P. H. (1994). Legal Rights for students with ADD. In P. Quinn (Ed.). ADD and the College Student: A Guide for High School and College Students With Attention Deficit Disorder [ADD y el estudiante universitario: Una guía para estudiantes de secundaria y universitarios con trastorno por déficit de atención] (pp. 139-148). Washington, DC: Magination Press and American Psychological Associtation.

Mc Comick, A. (1994). Learning accomodations at college. In P. Quinn (Ed.). ADD and the College Student: A Guide for High School and College Students With Attention Deficit Disorder [ADD y el estudiante universitario: Una guía para estudiantes de secundaria y universitarios con trastorno por déficit de atención] (pp. 123-130). Washington, DC: Magination Press and American Psychological Associtation.

Martinussen, R., Tannock, R., Chaban, P. (2011). Teachers' Reported Use of Instructional and Behavior Management Practices for Students with Behavior Problems: Relationship to Role and Level of Training in ADHD [Maestros reportan uso de prácticas instruccionales y del comportamiento en los estudiantes con problemas de comportamiento: relación entre función y nivel de entrenamiento en el déficit de atención e hiperactividad]. Child Youth Care Forum, 40, 193-210.

Nadeau, K. G. (1996). Adventure in Fast Forward. Life, Love, and Work for the ADD Adult [Aventuras adelantadas. Vida, amor y trabajo para el adulto con déficit de atención e hiperactividad]. New York: Brunner Routledge.

Neuwirth, S. (1994). Attention Deficit Hyperactivity Disorder [Trastorno hiperactivo de déficit de atención]. Betheseda: National Institutes of Health.

Ortiz, I. E., Sáenz, S., Escalona, P. R. y Vargas, M. A. (1996). Trastorno por déficit de atención con hiperactividad en niños y adultos. Medico Interamericano, 15, 535-538

O'Connor, K. (1994). Looking at collage programs. In P. Quinn (Ed.). ADD and the College Student: A Guide for High School and College Students With Attention Deficit Disorder [ADD y el estudiante universitario: Una guía para estudiantes de secundaria y universitarios con trastorno por déficit de atención]. (pp.67-73). Washington, DC: Magination Press and American Psychological Associtation.

Quesada, G. y Saenz, Y. (2009): Adult with ADHD (Adulto con déficit de atención e hiperactividad). Recuperado de http://www.univision.com/content/enfermedades 
Ramsey, S. (2009). ADHD Medication can Help Children Perform at School [La medicina para el déficit de atención e hiperactividad puede ayudar el rendimiento en la escuela]. Recuperado de http://www.topnews.us/ content/25017-adhd-medication-can-help-children-perform-better-school

Rush, C. y Harrison, P. (2008). Ascertaining Teachers' Perceptions of Working with Adolescents Diagnosed with Attention-Deficit/Hyperactivity Disorder [Percepción asertiva de maestros que trabajan con adolescentes diagnósticados con el desorden del déficit de atención e hiperactividad]. Educational Psychology in Practice, 24(3), 207-223.

Trampush, J. W., Miller, C. J., Newcorn, J. M. y Halperin, J. M. (2009). The Impact of Childhood ADHD on Dropping out of High School in Urban Adolescents/Young Adults [El impacto del déficit de atención e hiperactividad en la niñez en las bajas de la escuela superior en adolescentes/jóvenes adultos urbanos]. Journal Attention Disorder, 13(2), 127-136.

Searight, H. R. (2000). Adult ADHD: Evaluation and Treatment in Family Medicine [Adultos con déficit de atención e hiperactividad: evaluación y tratamiento en la medicina de familia].Am Fam Physician, 62(9):2077-2086. Recuperado de https://www.aafp.org/afp/2000/1101/p2077.html

Zeigler, C. A. (1995) Teanegers with ADD: A Parent's Guide. Massachusetts, MA; Woodbine House

\section{Anexo 1}

\section{Cuestionario a maestros y maestras}

1. ¿Qué métodos usted utiliza para impartir la enseñanza a sus estudiantes?

2. ¿Hace usted algunos cambios en su método de enseñanza-aprendizaje cuando identifica algún problema de aprendizaje o conducta en sus estudiantes? Explique.

3. ¿Ha escuchado hablar sobre el déficit de atención e hiperactividad (ADHD) en personas jóvenes adultas? ¿Como qué?

4. En la universidad/colegio o vocacional donde usted labora como profesor/ra, ¿ha encontrado estudiantado joven matriculado con esta condición?

5. ¿Es su universidad/colegio o vocacional un lugar apto o seguro para estos grupos de jóvenes con $\mathrm{ADHD}$ en referencia a la calidad educativa que estos imparten? Explique.

6. Como profesional de educación, ¿̇cuánto conoce del tema del ADHD en las personas adultas? ¿Qué ha escuchado?

7. Durante el periodo que usted se ha desarrollado como profesor/ra, ¿ha notado esta condición en sus estudiantes?

8. Estos o estas jóvenes que usted identifica como posibles personas candidatas a padecer el ADHD, ¿conocen su problema? Si la respuesta es sí, ¿desde cuándo tienen este conocimiento?

Si es no, ¿cómo usted les hace llegar la información pertinente para que se percaten de sus síntomas y puedan recibir ayuda?

9. ¿Sabe usted o tiene algún conocimiento de cuantos estudiantes con la condición del ADHD en su centro de trabajo reciben ayuda? ¿Qué clase de ayuda?

10. Actualmente, en su salón de clases, ¿̇iene jóvenes con la condición del ADHD?

11. ¿Qué métodos de enseñanza utiliza o utilizó para hacer factible el aprendizaje en jóvenes con $\mathrm{ADHD}$ ?

12. ¿Qué métodos de evaluación académicas utiliza con este tipo de estudiante?

13. ¿Toma o ha tomado algún tipo de curso o seminario que esté enfocado en cómo educar a las personas jóvenes adultas con ADHD en centros destinados a ofrecer estudios superiores? ¿Qué aprendió?

14. ¿Conoce o tiene idea de cuales políticas son ejercidas en su centro/colegios o universidad 
en relación con estos grupos de jóvenes? Describa brevemente.

15. ¿Conoce o tiene idea de las leyes que protegen a jóvenes con ADHD en los centros de estudios superiores? ¿Podría mencionarlas?

16. ¿Cuál es su opinión en relación con el trato educativo que reciben jóvenes con ADHD?

17. ¿Cree usted que se está haciendo lo pertinente para que estos grupos de jóvenes reciban igualdad universitaria? Explique.

18. ¿Considera usted que las universidades se encuentran preparadas con profesorado educado para llevar la enseñanza a estos grupos de estudiantes a través de métodos adecuados que les permitan expandir sus conocimientos? Explique.

19. ¿Qué recomendaciones haría usted en relación con el método de enseñanza impartido a estudiantes con ADD o ADHD?

20. Si usted no tiene suficiente conocimiento sobre el tema tratado o nunca lo ha escuchado, ¿̇cuál es su reacción luego de contestar este cuestionario sobre estudiantes que cursan estudios superiores con $\mathrm{ADD}$ o $\mathrm{ADHD}$ ?

21. ¿Qué efectos o cambios se produjeron en usted al conocer que puede tener este alumnado en su salón de clases?

22. ¿Qué medidas toma o tomará para lograr que su clase sea eficaz para jóvenes con ADHD?

23. ¿Está de acuerdo o se siente conforme con el sistema educativo que impera o por el que se rige su centro de trabajo? ¿Por qué?

24. Si pudiera lograr un cambio, ¿Qué cosas cambiaría en su centro de trabajo y por cuáles las sustituiría? ¿Por qué?

25. ¿Considera que su cultura es una influencia en su estilo de impartir la educación superior? ¿En qué aspecto? Explique.

26. ¿Ha padecido alguna vez depresión laborar? ¿Cuál fue la causa?

27. ¿Cree o considera usted que algún tipo de enfermedad o depresión laboral pueda afectar la enseñanza educativa que reciben las personas adultas universitarias? ¿Por qué?

\section{Cuestionario para diagnósticar el déficit de atención e hiperactividad (DSM-IV)}

\section{Atención}

¿Has experimentado dificultades con algunas de las siguientes, en más de un escenario (escuela, hogar, sociedad) y por más de 6 meses?

Escala de autodetección para personas adultas (frecuencias). Donde $\mathbf{1}$ es Nunca y $\mathbf{5}$ es Muy frecuente Nunca (1), Raramente (2), A veces (3), Frecuentemente (4), Muy frecuentemente (5)

10. ¿Luchas por mantener la atención a tareas o juegos?

11. ¿A menudo te descuidas haciendo problemas escolares?

12. ¿A menudo pierdes cosas necesarias de tarea o actividades? (ej. libros)

13. ¿A menudo tienes dificultad para realizar tareas o actividades?

14. ¿A menudo evitas o te desagrada ocuparte en tareas que requieren mucho esfuerzo mental?

15. ¿A menudo pareces no estar escuchando cuando te hablan directamente?

16. ¿Encuentras dificultades en seguir instrucciones y dejas de terminar una tarea?

17. ¿A menudo eres olvidadizo en tus tareas diarias?

18. ¿Te distraes fácilmente? 


\section{Hiperactividad/Impulsividad}

¿Has experimentado dificultad con algunas de las siguientes situaciones en más de un escenario (escuela, hogar, sociedad) y por más de 6 meses?

21. ¿A menudo juegas con las manos o los pies o te retuerces en tu asiento?

22. ¿A menudo dejas el asiento en clases o en otra situación donde se espera se permanezca sentado/a?

23. ¿A menudo corres o saltas excesivamente en situaciones donde es inapropiado?

24. ¿A menudo tienes dificultad jugando o trabajando en actividades pasivas?

25. ¿A menudo actúas como si fueras conducido/a por una moto?

26. ¿A menudo contestas sin pensar una pregunta que no ha estado completada?

27. ¿A menudo interrumpes o te introduces en otras conversaciones? (entremeterse en conversaciones o juegos)

28. ¿A menudo tienes dificultad de esperar tu turno?

29. ¿A menudo hablas excesivamente?

\section{BY-NC-ND}

